\title{
Zinc supplementation in children with cholera in Bangladesh: randomised controlled trial
}

\author{
S K Roy, senior scientist, M Jahangir Hossain, assistant scientist, Wajiha Khatun, research officer, \\ Barnali Chakraborty, research officer, S Chowdhury, research physician, Afroza Begum, research \\ assistant, Syeda Mah-e-Muneer, research assistant, Sohana Shafique, research investigator, \\ Mansura Khanam, research assistant, R Chowdhury, research physician
}

International Centre for Diarrhoeal Disease Research, Bangladesh (ICDDR, B), 68, Shaheed Tajuddin Ahmed Sarani, Mohakhali, Dhaka -1212, Bangladesh

Correspondence to: S K Roy skroy@icddrb.org

doi:10.1136/bmj.39416.646250.AE

\section{ABSTRACT}

Objective To investigate the impact of zinc supplementation in children with cholera.

Design Double blind, randomised, placebo controlled trial.

Setting Dhaka Hospital, Bangladesh.

Participants 179 children aged 3-14 years with watery stool volume in cholera. diarrhoea and stool dark field examination positive for Vibrio cholerae and confirmed by stool culture.

Intervention Children were randomised to receive $30 \mathrm{mg}$ elemental zinc per day $(n=90)$ or placebo $(n=89)$ until recovery. All children received erythromycin suspension orally in a dose of $12.5 \mathrm{mg} / \mathrm{kg}$ every six hours for three days. Main outcome measures Duration of diarrhoea and stool output.

Results 82 children in each group completed the study. More patients in the zinc group than in the control group recovered by two days ( $49 \%$ v $32 \%, \mathrm{P}=0.032)$ and by three days $(81 \% \vee 68 \%, P=0.03)$. Zinc supplemented patients had $12 \%$ shorter duration of diarrhoea than control patients $(64.1 \vee 72.8 \mathrm{~h}, \mathrm{P}=0.028)$ and $11 \%$ less stool output (1.6 v $1.8 \mathrm{~kg} /$ day, $\mathrm{P}=0.039)$.

Conclusion Zinc supplementation significantly reduced the duration of diarrhoea and stool output in children with cholera. Children with cholera should be supplemented with zinc to reduce its duration and severity.

Trial registration Clinical trials NCT00226616.

\section{INTRODUCTION}

Cholera is caused by infection of the small intestine by Vibrio cholerae 01 and 0139 , and the disease is characterised by massive watery diarrhoea, vomiting, dehydration, and metabolic acidosis, leading to death if not efficiently managed. ${ }^{1}$ It is the most severe form of all secretory diarrhoea in many parts of the world. As cholera is a disease associated with high mortality, treatment has been directed to lifesaving approaches, including rapid correction of fluid and electrolyte deficits. Various interventions have been tested to reduce stool volume and duration of diarrhoea, including antisecretory agents such as chlorpromazine, and none was found to be clinically useful. Antimicrobials effective against $\mathrm{V}$ cholerae are useful in shortening the duration of diarrhoea and reducing

In 1974 the genetic syndrome of zinc deficiency, acrodermatitis enteropathica, which manifests as skin lesions and chronic diarrhoea, was seen to improve rapidly after zinc supplementation..$^{2}$ In children with persistent diarrhoea, the loss of zinc in stool was reported to be as high as $300 \mu \mathrm{g} / \mathrm{kg} / \mathrm{day} .^{3}$ Zinc supplementation in children with acute diarrhoea has been found to be associated with a $28 \%$ reduction in stool volume and a 33\% reduction in the duration of recovery from persistent diarrhoea. ${ }^{45}$ The earlier recovery from diarrhoea and reduced stool output could be related to the better repair of mucosal integrity or improved transport of water and electrolytes with zinc supplementation. ${ }^{6}$ A pooled analysis of studies of zinc supplementation in children in different countries showed similar beneficial effects of zinc in acute and persistent diarrhoea, ${ }^{7}$ but the impact of zinc on severe diarrhoea (such as in cholera) is not known. An experimental study found that net water and sodium secretion induced by cholera toxin was four times greater in zinc deficient animals than in animals fed unlimited zinc; in zinc deficient animals a $40 \%$ reduction in secretion was seen after a 48 hour zinc repletion. ${ }^{8}$ Many studies have shown beneficial effects of zinc supplementation in children under 3 years old with diarrhoea, ${ }^{4579}$ but the effect of zinc in older age groups has not been studied.

Zinc may reduce the severity and duration of diarrhoea caused by cholera in children, which will help them to return home sooner and free up beds for other children with diarrhoea. The reduction of disease burden would reduce expenditure for hospitals and families. We investigated the impact of zinc on severe diarrhoea caused by cholera in children aged 3-14 years, as they are at the highest risk of contracting cholera in countries such as Bangladesh in which cholera is endemic.

\section{METHODS}

We did a randomised, double blind, placebo controlled clinical trial at the Dhaka Hospital of the International 
Table 1| Baseline characteristics of study children. Values are mean (SD) unless stated otherwise

\begin{tabular}{lccc} 
Characteristics & Zinc (n=90) & Control (n=89) & P value \\
Age (months) & $81.5(36.5)$ & $83.0(36.5)$ & 0.790 \\
\hline Sex (No male/No female) & $62 / 28$ & $51 / 38$ & 0.108 \\
\hline Weight (kg) & $17.3(6.1)$ & $16.7(5.1)$ & 0.472 \\
\hline Weight for age median (\% of NCHS median) & $74.9(11.1)$ & $71.7(8.9)$ & 0.033 \\
\hline Height (cm) & $109(17.1)$ & $107(20.3)$ & 0.548 \\
\hline Body mass index & $17.5(6.2)$ & $16.7(5.1)$ & 0.363 \\
\hline Duration of diarrhoea before admission (hours) & $10.5(5.8)$ & $10.0(5.3)$ & 0.564 \\
\hline Stool volume during four hours of observation (ml) & $767(423)$ & $774(468)$ & 0.912 \\
\hline Time for rehydration (hours) & $4.7(0.86)$ & $4.8(0.91)$ & 0.429 \\
\hline Dehydration status: & & $78(88)$ & 0.111 \\
\hline No (\%) severe & $85(94)$ & $11(12)$ & \\
\hline No (\%) some & $5(6)$ & & \\
\hline NCHS=National Centre for Health and Statistics. & & \\
\hline
\end{tabular}

Centre for Diarrhoeal Disease Research, Bangladesh between November 2000 and June 2002.

\section{Study participants}

Eligible patients were children of both sexes, aged 3-14 years, admitted to the hospital with cholera within 24 hours of onset, who had some dehydration or severe dehydration according to World Health Organization criteria,${ }^{10}$ had a high purging rate (stool output more than $4 \mathrm{ml} / \mathrm{kg} /$ hour for the first six hours of observation after initial rehydration), and had positive dark field examination of stool (a direct observation of the movement of $V$ cholerae against a dark background under high power in a light microscope). We excluded patients with any of the following criteria: unable to rehydrate within six hours, negative dark field examination, systemic illness

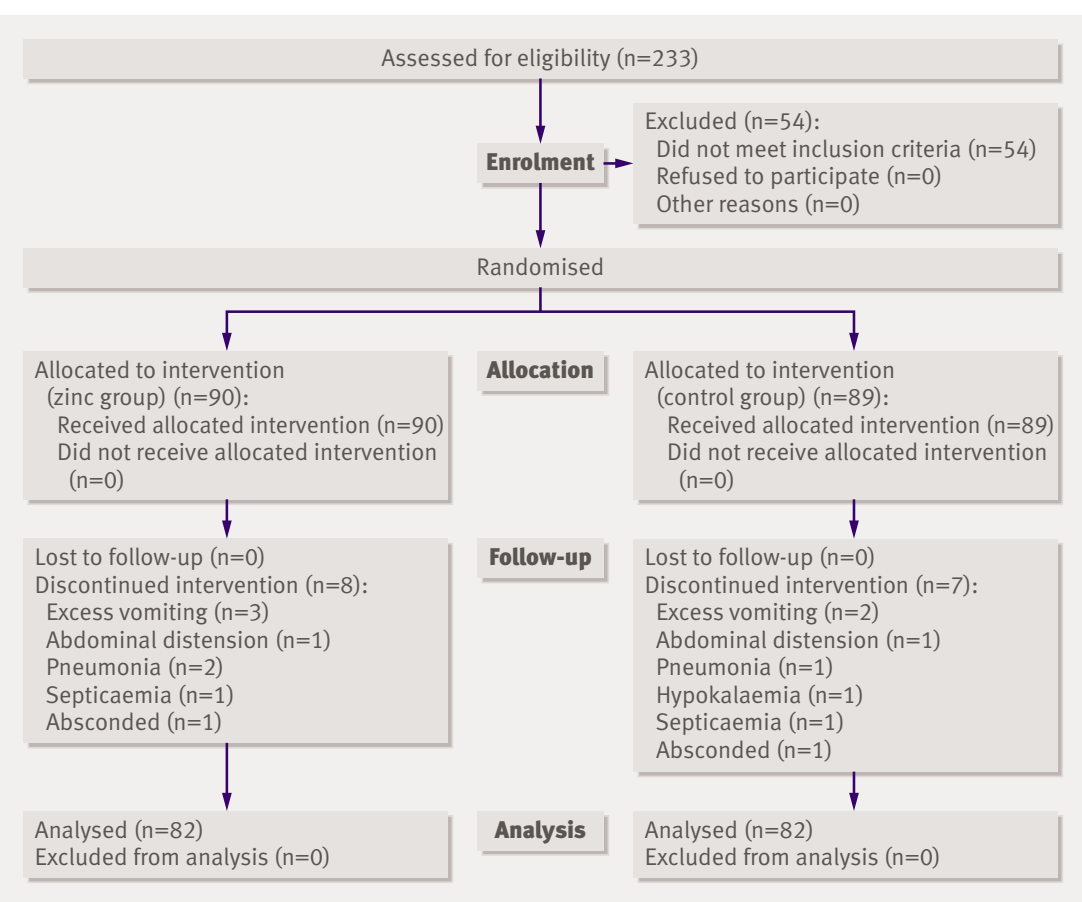

Fig 1 Trial profile requiring immediate administration of antibiotics, receipt of antibiotics within 24 hours before admission to hospital, presented with bloody mucoid diarrhoea, unconscious, or had medical emergencies. The parents or guardians/caregivers of each patient gave written informed consent before enrolment into the study. We estimated the sample size needed to detect a $20 \%$ difference in the duration of illness between the zinc group and control group at 90\% power and 5\% level of confidence to be 78 in each group. ${ }^{11}$ Considering the $15 \%$ drop out according to Kirkwood, the total sample size was $180 .{ }^{12}$

\section{Study groups and randomisation}

We assigned patients to the study groups when they met the criteria for selection and recruited patients into the study when cholera was confirmed by stool culture. We used a block randomisation with a block length of six. We randomised enrolled children into two groups: those in the intervention group received zinc supplementation and those in the control group received a placebo for zinc; all children received erythromycin. For double blinding, we used identical bottles containing syrup of same colour and flavour with or without zinc and labelled the bottles only with the random number previously allocated to one of the two interventions.

\section{Intervention}

Patients in the zinc group received $30 \mathrm{mg}$ of elemental zinc as acetate each day in two equally divided doses, and those in the control group received placebo, until resolution of diarrhoea or for up to seven days. Base syrup was used as placebo for patients randomised to the control group, and zinc was added to this base syrup for patients randomised to the zinc group. The base syrup used for both intervention groups was of the same chemical composition. Cost of zinc treatment was $\$ 0.14(£ 0.07$; €0.10) for three days. All children received erythromycin in a dose of $12.5 \mathrm{mg} / \mathrm{kg}$ every six hours for three days (total of 12 doses). We used the 
disc diffusion method to test the sensitivity of $\mathrm{V}$ cholerae to erythromycin.

\section{Clinical procedure}

After screening for positive dark field results, we corrected dehydration with intravenous fluid, oral fluid, or both. Patients were rehydrated with intravenous polyelectrolyte solution (Dhaka Solution with $133 \mathrm{mmol} / 1$ sodium, $13 \mathrm{mmol} / 1$ potassium, $98 \mathrm{mmol} / 1$ chloride, and $48 \mathrm{mmol} / \mathrm{l}$ acetate) or rice based oral rehydration salts solution (with $90 \mathrm{mmol} / \mathrm{l}$ sodium, $20 \mathrm{mmol} / \mathrm{l}$ potassium, $80 \mathrm{mmol} / \mathrm{l}$ chloride, and $30 \mathrm{mmol} / \mathrm{l}$ bicarbonate), depending on their dehydration status and according to WHO guidelines. From the start of the study, we measured intakes of oral rehydration salts and fluids and output of stool and urine eight hourly until resolution of diarrhoea. Study physicians observed stools for watery, liquid, soft, or formed consistency, with daily validation by the investigators (SKR and JMH). All children had a standardised hospital diet, which included milk sujee (rice powder boiled in milk with added sugar), rice, chicken or fish, vegetables, dal (lentils), milk, bread, and banana. We cultured stools for isolation of $\mathrm{V}$ cholerae.

We withdrew patients from the study if they developed complications such as excessive vomiting, abdominal distension, pneumonia, hypokalaemia, or septicaemia or left the hospital. In some patients who had excessive vomiting, oral rehydration salts had to be stopped and intravenous fluids given to correct dehydration. Patients with abdominal distension due to ileus were kept nil by mouth and transferred to the special care unit for appropriate treatment with other antibiotics. Patients who developed hypokalaemia, pneumonia, or septicaemia were also transferred to the special care unit. These patients who dropped out could not follow the study protocol; we included them in the intention to treat analysis. The end point for these patients was drop-out rather than clinical recovery. The remaining patients followed the full procedure of the study to reach the end points, and we included them in the per protocol analysis.

\section{Laboratory investigation}

Owing to resources limitations, we collected $2 \mathrm{ml}$ of venous blood from every alternate randomised child for estimation of serum zinc on admission and on day 10 after the start of the intervention. We used an atomic

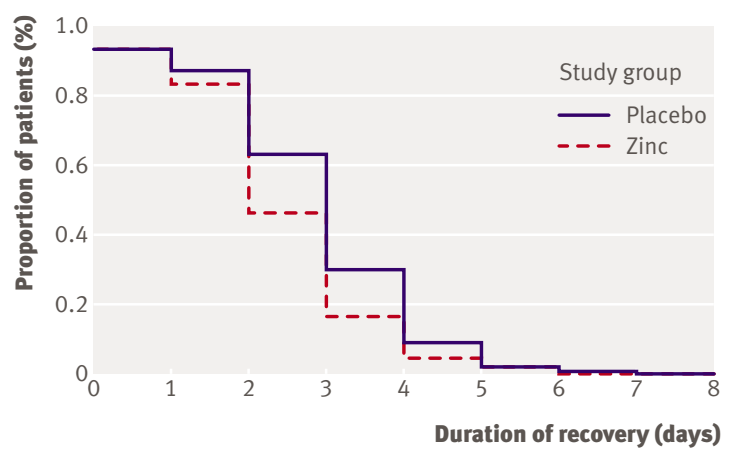

Fig 2 Probability of having diarrhoea by day of intervention. Kaplan-Meier survival curve in intention to treat analysis, showing recovery from diarrhoea in zinc supplemented group $(n=90)$ and control group $(n=89)$, without drop-out. Median recovery 2 days in zinc supplemented group and 3 days in control group ( $\log$ rank test, $\mathrm{P}=0.024)$

absorption spectrophotometer (Pye Unicam, SP9) to measure serum zinc. ${ }^{4} \mathrm{We}$ collected urine separately by the application of paediatric urine collection bags. We measured stool weight with a pre-weighed bucket into which stool was collected directly by means of a cholera cot with plastic sheets draining stool directly to the bucket. The weight of the stool was measured in a Toledo Scale (Toledo, USA) with a precision of $1 \mathrm{~g} .{ }^{45}$ We started measurement of stool immediately after recruitment into the study and stopped when the last liquid stool was passed.

\section{Anthropometric measurements}

We used body weight after initial rehydration to determine nutritional status. We measured body weights every day between 9 am and 11 am during the hospital stay; we measured heights once at discharge after recovery rather than on admission when patients were unable to stand correctly. We calculated the nutritional status of the children as a percentage of the National Centre for Health and Statistics median.

\section{Study outcomes}

The primary outcomes were diarrhoeal stool output and time to resolution of diarrhoea (defined as the time from the start of rehydration and collection of stool until the first formed stools or no stool for 24 hours; every eight hour period of measuring stool output was counted for defining the recovery period). Secondary

$\overline{\text { Table } 2 \text { | Impact of zinc supplementation on clinical outcomes in children with cholera. Values are mean (95\% confidence interval) }}$ unless stated otherwise

\begin{tabular}{|c|c|c|c|}
\hline Variables & Zinc $(n=82)$ & Control $(n=82)$ & $P$ value \\
\hline Duration of diarrhoea (hours) & 64.1 (58.5 to 69.7$)$ & $72.8(67.4$ to 78.6$)$ & 0.028 \\
\hline Total stool weight (kg/day) & $1.6(1.4$ to 1.7$)$ & 1.8 (1.7 to 2.0$)$ & 0.039 \\
\hline Total vomitus weight (g) & 789.9 (585.97 to 993.7$)$ & 565.1 (359.6 to 770.7 ) & 0.126 \\
\hline Total ORS intake (ml) & 706.9 (609.9 to 804.0$)$ & 693.9 (595.9 to 791.9 ) & 0.852 \\
\hline
\end{tabular}


Table 3 Impact of zinc supplementation on serum zinc concentration. Values are mean (SD) unless stated otherwise

\begin{tabular}{|c|c|c|c|}
\hline \multirow[b]{2}{*}{ Variables } & \multicolumn{2}{|c|}{ Serum zinc concentration $(\mathrm{mg} / \mathrm{dl})$} & \multirow[b]{2}{*}{$P$ value } \\
\hline & On admission after rehydration & At day 10 & \\
\hline Zinc $(n=41)$ & $0.58(0.11)$ & $0.73(0.16)^{\star}$ & 0.001 \\
\hline Control $(n=43)$ & $0.55(0.11)$ & $0.64(0.12)$ & 0.001 \\
\hline
\end{tabular}

outcome measures were the serum zinc status and the intake of oral rehydration salts or intravenous fluid.

\section{Statistical analysis}

The investigators checked data daily at recording, cleaned them, and then entered them on a personal computer with SPSS/PC+. The consistency check used logical programs. We tested normally distributed continuous data for comparison of group means by using Student's t test. We used Kaplan-Meier survival analysis to compare differences in the time to resolution of diarrhoea between the two groups and the $\log$ rank test to test for significance. We used $\chi 2$ to compare proportions. We considered differences to be significant at the $5 \%$ probability level.

We analysed the probability of having diarrhoea on an intention to treat basis, including all patients assigned to the trial. We did Kaplan-Meier survival analysis on intention to treat and per protocol bases, to see the effect of zinc with and without the dropout cases. We analysed outcome variables (duration of diarrhoea, total stool weight, and serum zinc concentration) only for patients who completed the study.

\section{RESULTS}

Enrolment of study participants

During the enrolment period, 233 patients were screened as having cholera (fig 1). Of these, we recruited 179 patients who met the eligibility criteria and gave consent to take part in the trial. We assigned 90 children to zinc supplements and 89 to the control group. Eight children from the zinc group and seven from the control group were withdrawn from the study

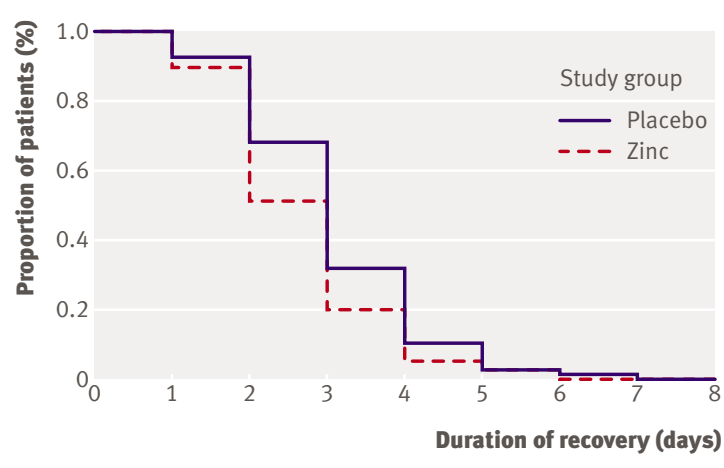

Fig 3 Probability of having diarrhoea by day of intervention. Kaplan-Meier survival curve in per protocol analysis, showing recovery from diarrhoea in zinc supplemented group $(n=82)$ and control group $(n=82)$, with drop-out. Median recovery 2 days in both groups (log rank test, $\mathrm{P}=0.032$ ) because of the development of complications such as excessive vomiting $(\mathrm{n}=5)$, abdominal distension $(\mathrm{n}=2)$, pneumonia $(n=3)$, hypokalaemia $(n=1)$, or septicaemia $(n=2)$ or because they left the hospital $(n=2)$. Finally, 164 children (82 in each group) completed the study. These patients could follow the study protocol and procedure and could reach the end points, and we included them in the per protocol analysis. Out of the 164 patients, 70\% were over 5 years of age.

All patients were culture positive for V cholerae on day $1,44(27 \%)$ were positive on day $2,25(15 \%)$ were positive on day 3 , and none was positive on day 4 . Culture negative but dark field positive patients were not included. All of the Vcholerae cultures were sensitive to erythromycin.

\section{Baseline characteristics}

Baseline characteristics such as age, sex, dehydration status on admission, mean duration of diarrhoea before enrolment, and time taken for initial rehydration were comparable between the two groups (table 1). On recruitment, $90 \%$ of the children were severely dehydrated, of whom $48 \%$ were randomised to zinc and $52 \%$ to the control group $(\mathrm{P}=0.111)$. The syrup, with or without zinc, was well tolerated by the children. Vomiting was recorded and did not differ significantly between the groups.

\section{Primary outcomes}

Time to resolution of diarrhoea-In the intention to treat analysis, the proportions of children who clinically recovered from cholera by day $2(49(54 \%)$ v 33 $(37 \%), \mathrm{P}=0.024)$ and by day $3(75(83 \%)$ v $62(70 \%)$, $\mathrm{P}=0.024)$ were significantly higher in the zinc supplemented children (fig 2). The median time until recovery was shorter among the zinc supplemented children than among the children in the control group ( $2 \mathrm{v} 3$ days, $\mathrm{P}=0.032$ ). In the per protocol analysis, 40 $(49 \%)$ children in the zinc supplemented group and 26 $(32 \%)$ children in the control group recovered by two days of treatment $(\mathrm{P}=0.032) ; 66(81 \%)$ zinc supplemented children and $56(68 \%)$ children in the control group recovered by three days $(\mathrm{P}=0.032)$ (fig 3$)$. The mean duration of recovery was significantly shorter among the zinc supplemented children than among the children in the control group (2.68 v 3.06 days, $\mathrm{P}=0.032$ ).

Duration of diarrhoea-Children who received zinc had a $12 \%$ shorter mean duration of diarrhoea $(64.1 \mathrm{v}$ 72.8 hours, $\mathrm{P}=0.028$ ) than did those who did not receive zinc (table 2). 


\section{WHAT IS ALREADY KNOWN ON THIS TOPIC}

Zinc supplementation is beneficial in young children with diarrhoea

Beneficial effects of zinc on mild diarrhoea have been seen in a community setting

\section{WHAT THIS STUDY ADDS}

Zinc supplementation decreased stool volume and duration of recovery in children aged 3-14 years

Zinc supplementation was effective in severe diarrhoea caused by cholera

Stool output-We found an $11 \%$ reduction in diarrhoeal stool weight in the zinc group compared with the control group (1.6 v $1.8 \mathrm{~kg}, \mathrm{P}=0.039)$ (table 2).

Rate of vomiting - The mean total weight of vomitus was comparable between the zinc group and the control group (789.9 v565.1 g, $\mathrm{P}=0.126$ ) (table 2).

\section{Secondary outcomes}

Serum zinc-Serum zinc significantly increased from baseline to recovery in children in both groups. We calculated the zinc content of the hospital diet, and it was comparable between the groups. The mean dietary zinc concentration was $8.6 \mathrm{mg} /$ day, and zinc came from foods such as rice, fish, chicken, bread, and milk. However, the net increase in serum zinc concentration in the zinc supplemented group was significantly higher $(26 \%)$ than that $(16 \%)$ in the control group $(\mathrm{P}<0.001)$ (table 3).

Oral rehydration salts intake-The mean intake of oral rehydration salts during diarrhoea did not differ between the two study groups $(706.9$ v $693.9 \mathrm{ml}$, $\mathrm{P}=0.852$ ) (table 2).

\section{DISCUSSION}

We saw a significant reduction in both the duration and severity of diarrhoea with zinc supplementation. Our earlier studies with zinc supplementation in acute and persistent diarrhoea without cholera in younger patients showed benefits in terms of reduction in stool output and duration of diarrhoea. ${ }^{457}$ This study showed enhanced clinical recovery and reduced stool output from cholera in zinc supplemented older children.

Our study has shown for the first time the effect of zinc on severe diarrhoea caused by cholera in older children (3-14 years). We found significantly reduced stool output and faster recovery in the zinc supplemented children. In this clinical trial with continuous observation, patients were closely monitored, stool weight was measured, and the cause of diarrhoea was confirmed by stool culture. Patients with zinc supplementation did not have excess vomiting compared with the control group. Our results showed an additional benefit of zinc over that of antibiotics in children with cholera.
Pooled analyses of data from zinc trials in acute and persistent diarrhoea in developing countries showed that zinc, given in a daily dose of about twice the recommended daily amount, significantly reduced the duration of acute and persistent diarrhoea (less severe diarrhoea than in this study). ${ }^{7}$ Zinc has been shown to reduce the duration of recovery in children who had low rectal mucosal zinc and low serum zinc concentration. ${ }^{13}$ Compared with an $8-21 \%$ reduction in the duration of recovery in patients with acute diarrhoea not admitted to hospital, the $12 \%$ reduction in duration of cholera in our study is encouraging. ${ }^{7}$ Earlier recovery from cholera would reduce the load on hospitals in countries where cholera is endemic, as well as reducing the disease burden with its economic implications.

Our study shows the effect of zinc on severe diarrhoea, an effect comparable to that seen in other studies. The pooled analysis of the results of zinc supplementation studies showed a $15 \%$ reduction in duration of diarrhoea in Indonesia, India, and Bangladesh. ${ }^{7}$ Our results indicate that zinc might have a lifesaving impact in cholera epidemics.

The results show enhanced recovery and reduced stool weight from severe diarrhoea caused by cholera. The exact mechanism by which zinc works is not known, but the following can be considered: zinc might work through improved electrolyte transport and water absorption, ${ }^{8}$ it has an impact on immunity to limit bacterial proliferation, and it has been shown to improve mucosal permeability. ${ }^{14-17}$ Zinc could be added to treatment policies for life threatening diarrhoea, as in cholera.

In our study, zinc supplementation reduced the average duration of diarrhoea by around eight hours. This can be considered important for resource limited countries such as Bangladesh, as children can be released earlier from hospital and beds can be freed for other patients. Children can go back to their schools earlier. It also reduces the treatment cost for the hospital and families.

We recommend the following areas of further research: the mechanism of action of zinc in diarrhoea, the impact of zinc supplementation in adult diarrhoea such as that caused by cholera or enterotoxigenic Escherichia coli, the impact of zinc supplementation during cholera epidemics, and improving the strategy of zinc supplementation in diarrhoea by adding zinc to oral rehydration solution.

Contributors: SKR contributed to the design of the study, secured funding, supervised patient management and clinical evaluation, and provided substantial advice on data analysis and critical interpretation of results. $\mathrm{MJH}$ contributed to the design of the study, and supervised patient management, clinical evaluation, and writing the manuscript. WK contributed to analysis and interpretation of data and writing the manuscript. BC, SC, AB, SMM, SS, MK, and RC were involved with interpreting the results and writing the manuscript. SKR is the guarantor Funding: World Bank grant for nutrition centre of excellence at ICDDR,B and Thrasher Research Fund, USA. The funding sources had no role in study design, data collection, analysis, interpretation of data, or in writing this report.

Competing interests: None declared.

Ethical approval: Ethical review committee of ICDDR, B. 
Provenance and peer review: Not commissioned; externally peer reviewed.

1 Rabbani GH, Sack DA, Choudhury MR. Vibrio cholerae. In: Militoes MD, Bier JW, eds. International handbook of food borne pathogens. New York, Basel: Marcel Dekker, 2003:217-35.

2 Moynehan EJ. Acrodermatitis enteropathica: a lethal inherited human zinc deficiency. Lancet 1974;2:399-400.

3 Rothbaum RJ, Maur PR, Farrell MK. Serum alkaline phosphate and zinc under nutrition in infants with chronic diarrhoea. Am J Clin Nutr 1982;35:595-8.

4 Roy SK, Tomkin AM, Akramuzzaman SM, Behrens RH, Haider R, Mahalnabis D, et al. Randomized controlled trial of zinc supplementation in malnourished Bangladeshi children with acute diarrhoea. Arch Dis Child 1997;77:196-200.

5 Roy SK, Tomkin AM, Mahalnabis D, Akramuzzaman SM, Haider R, Behrens RH, et al. Impact of zinc supplementation on persistent diarrhoea in malnourished Bangladeshi children. Acta Paediatr 1998;87:1235-9.

6 Roy SK, Behrens RH, Haider R, Akramuzzaman SM, Mahalnabis D, Wahed MA, et al. Impact of zinc supplementation on intestinal permeability in Bangladeshi children with acute diarrhoea and persistent diarrhoea syndrome. J Pediatr Gastroenterol Nutr 1992;15:289-96.

7 Bhutta ZA, Bird SM, Black RE, Brown KH, Gardner JM, Hidayat A, et al. Therapeutic effects of oral zinc in acute and persistent diarrhoea in children in developing countries: pooled analysis of randomized controlled trials. Am J Clin Nutr 2000;72:1516-22.

8 Roy SK, Tomkins AM, Ara G, Jolly SP, Khatun W, Chowdhury R, et al. Impact of zinc deficiency on Vibrio cholerae enterotoxin-stimulated water and electrolyte transport in animal model. J Health Popul Nutr 2006;24:42-7.
9 Dutta P, Mitra U, Datta A, Niyogi SK, Dutta SA, Manna B, et al. Impact of zinc supplementation in malnourished children with acute watery diarrhoea. J Trop Pediatr 2000;46:259-63.

10 World Health Organization. The treatment of diarrhoea: a manual for physicians and other senior health workers . Geneva: WHO, 2005:1-50.

11 Khan WA, Begum M, Salam MA, Bardhan PK, Islam MR, Mahalanabis D. Comparative trial of five antimicrobial compounds in the treatment of cholera in adults. Trans $R$ Soc Trop Med Hyg 1995;89:103-6.

12 Kirkwood B. Essentials of medical statistics.1st ed. Oxford: Blackwell Science, 1998:191-7.

13 Sachdev HPS, Mittal NK, Yadav HS. Oral zinc supplementation in persistent diarrhoea in infants. Ann Trop Paediatr 1990;10:63-9.

14 Roy SK, Behrens RH, Haider R, Akramuzzaman SM, Mahalanabis D, Wahed MA, et al. Impact of zinc supplementation on intestinal permeability in Bangladeshi children with acute diarrhoea and persistent diarrhoea and persistent syndrome.J Pediatr Gastroenterol Nutr 1992;15:289-96.

15 Albert MJ, Qadri F, Wahed MA, Ahmed T, Rahman ASMH, Ahmed F, et al. Supplementation with zinc, but not vitamin A improves seroconversion to vibriocidal antibody in children given oral cholera vaccine. J Infect Dis 2003;187:909-13.

16 Karlsen TH, Sommerfelt H, Klomstad S, Andersen PK, Strand TA, Ulvik RJ, et al. Zinc-a new adjuvant for mucosal immunization? In: 11th International Congress of Mucosal Immunology. Orlando, FL: Society of Mucosal Immunology, 2002.

17 Karlsen TH, Sommerfelt H, Klomstad S, Andersen PK, Strand TA, Ulvik RJ, et al. Intestinal and systemic immune responses to an oral cholera toxoid $B$ subunit whole-cell vaccine administrated during zinc supplementation. Infect Immun 2003;71:3909-13.

Accepted: 11 November 2007 